INVESTIGACIÓN

\title{
PLAN PROSPECTIVO Y ESTRATÉGICO PARA LA EMPRESA DE CONSTRUCCIÓN DE OBRAS CIVILES MOVITEC LTDA. AÑO 2023*
}

\section{PROSPECTIVE AND STRATEGIC PLAN FOR THE CONSTRUCTION COMPANY OF CIVIL WORKS MOVITEC LTDA. YEAR 2023}

\author{
Carlos Eduardo Niño Castellanos** \\ Benjamín Andrés Manjarrés Zárate***
}

Recibido: 10 de octubre de 2017

Evaluado: 20 de noviembre de 2017

Aprobado: 25 de enero de 2018

\section{RESUMEN}

La planeación estratégica basada en la prospectiva se constituye en una herramienta clave para las organizaciones en cuanto a la construcción de su futuro. Permite equilibrar el pensamiento estratégico, el deseo de visualizar un horizonte ideal, y los cursos de acción necesarios para alcanzar dicho derrotero. Las organizaciones de cualquier naturaleza, tipología, tamaño u objeto social, pueden aplicar esta metodología, a fin de mejorar su capacidad directiva, en lo concerniente a los procesos de planeación y control administrativo.

* Artículo de estudio.

** Ingeniero de Sistemas, egresado de la Universidad INCCA de Colombia. Especialista en formulación y evaluación de proyectos, Universidad Católica de Colombia. Maestrante en Administración de Organizaciones, Universidad Nacional Abierta y a Distancia (UNAD). Correo electrónico: cenino1998@yahoo.com

*** Administrador de Empresas, egresado de la Universidad de Cundinamarca. Especialista en Docencia, Universitaria Universidad Militar Nueva Granada. Maestrante en Administración de Organizaciones Universidad Nacional Abierta y a Distancia (UNAD). Correo electrónico: benjand83092295@gmail.com 
Movitec Ltda. es una empresa fundada hace 39 años, su objeto social es el desarrollo de productos y servicios asociados a la construcción e ingeniería de obras civiles. Tiene un desafío importante para el año 2023, el cual consiste en "ser la mejor" empresa del sector; por cuanto, a través de la aplicación de las herramientas prospectivas, se definió que el futurible ideal para consolidar sus ventajas competitivas, se basa en una mejor eficiencia y transparencia en cuanto a la adjudicación de concesiones de obras de infraestructura pública por parte del Estado, y el acceso a nuevas tecnologías ambientales, sociales y económicamente sostenibles, lo cual le permite a la compañía mejorar la eficiencia, eficacia y efectividad en sus procesos, y generar resultados importantes, en términos de valor agregado, de acuerdo con cada una de sus perspectivas: financiera, procesos internos, clientes, y aprendizaje y crecimiento.

Palabras clave: prospectiva estratégica, Movitec Ltda., ingeniería civil, variables, actores, escenarios.

\section{ABSTRACT}

The strategic planning based on prospective is a key tool for organizations in terms of building their future, because it allows balance strategic thinking, the desire to visualize an ideal horizon and the actions required to achieve that purpose. So, organizations of any kind, type, size or purpose, can apply this methodology in order to improve their management capacity, regarding the planning processes and management control.

Movitec Ltda., is a company founded 39 years ago and its purpose is the development of products and services associated with the construction and civil engineering works. Movitec Ltda., has a major challenge for the year 2023, which is to "Be the Best" company in the sector, because, through the application of prospective tools, it was decided that the ideal place to consolidate their future competitive advantages, is based on the probability of improved efficiency and transparency, achieving government contracts in public infrastructure and access to new environmental, social and economically sustainable technologies, allowing the company to improve efficiency, effectiveness and efficiency in its processes, and generating significant results in terms of value added, according to each of their perspectives: financial, internal processes, customer, learning and growth. Keywords: Strategic Foresight, Movitec Ltda., Civil Engineering, Variables, Actors, Scenarios. 


\section{INTRODUCCIÓN}

Según Godet (2009), la prospectiva estratégica se constituye en una herramienta fundamental para empresas y territorios, de acuerdo con las tendencias y riesgos de cambios abruptos, se cambia el presente y se interpela la estrategia para lograr un escenario futurible ideal. Por tanto, se articula en tres grandes procesos: 1) reflexión colectiva, que comprende el planteamiento del problema, diagnóstico de la empresa, identificación de variables internas y externas, análisis de la dinámica de la empresa en el entorno e identificación de opciones estratégicas; 2) preparación de la decisión, que consta de la evaluación de opciones estratégicas y jerarquización de los objetivos; y 3) la acción en la que se fijan planes operativos y su implementación (Godet, 2009, p. 31).

La investigación que se presenta en este artículo consta del desarrollo del proceso de planeación estratégica prospectiva, aplicado a la empresa Movitec Ltda. El objeto social de la empresa está encaminado hacia la producción y prestación de servicios de construcción e ingeniería civil de obras, teniendo como horizonte de prospección el año 2023. En primer lugar, se realiza un diagnóstico interno y externo de la compañía, para identificar los factores críticos de éxito; posteriormente, se aplica el método Delphi, con el propósito de identificar los factores de cambio o variables estratégicas que se analizan por medio del método MICMAC; se identifica el juego de actores mediante el método MACTOR, se diseñan los escenarios alternos y apuesta por medio del método de ejes cruzados de Peter Schwartz; se analizan las opciones estratégicas a través de la Matriz de Importancia y Gobernabilidad (IGO) de Francisco Mojica; y se estructuran los proyectos estratégicos necesarios acorde con las estrategias identificadas y el escenario apuesta.

De esta forma, la empresa Movitec Ltda. puede consolidar sus ventajas competitivas en el subsector de la construcción de obras civiles, tomando como base las tendencias a futuro del comportamiento de las variables estratégicas internas y externas, que giran en torno a la visualización del futuro ideal o futurible apuesta de la compañía. Por tal motivo, es importante reflexionar sobre las cinco preguntas fundamentales para construir el futuro ideal: ¿quién soy?, ¿qué puede ocurrir?, ¿qué puedo hacer?, ¿qué voy a hacer? y ¿cómo lo voy a hacer? (Godet, 2009, p. 23)

La empresa Movitec Ltda. es una compañía ubicada en la ciudad de Bogotá, se dedica desde hace 39 años al desarrollo de obras de construcción de tipo civil, ofreciendo los siguientes productos y servicios: movimiento de tierras, elaboración de concretos, construcción de pavimentos, diseño y construcción de estructuras 
metálicas para puentes, cerramientos y cubiertas, transporte de materiales y transporte de maquinaria.

El objetivo general de la presente investigación consiste en construir el plan prospectivo y estratégico para la empresa Movitec Ltda. hacia el año 2023. Los objetivos específicos se resumen en: comprender la situación actual de la compañía Movitec Ltda. por medio de un diagnóstico estratégico interno y externo, identificar los factores de cambio que inciden en la compañía Movitec Ltda. por medio del análisis estructural, analizar el impacto de los actores sociales que influyen en la compañía Movitec Ltda., construir los escenarios probables y escenarios alternos de la compañía Movitec Ltda., para el año 2023, utilizando las herramientas prospectivas pertinentes, y diseñar planes tácticos y operativos para la empresa Movitec Ltda., conforme al análisis estratégico y la aplicación de las herramientas prospectivas

\section{METODOLOGÍA}

El enfoque de investigación que se tomó en cuenta para el presente estudio fue mixto cuantitativo y cualitativo-, se buscó recopilar y analizar datos cuantitativos y cualitativos de forma integrada, sistémica y conjunta, a fin de establecer, describir y predecir el comportamiento de las variables estratégicas que inciden directa e indirectamente en la compañía Movitec Ltda. (Hernández, Fernández y Baptista, 2010, p. 546). De esta forma, se aplicaron técnicas e instrumentos de recolección de datos pertinentes para el análisis estadístico de los resultados obtenidos -encuestas, entrevistas y listas de verificación-, así como, técnicas cualitativas - método Delphi-, con el fin de planificar los escenarios apuesta y definir las estrategias necesarias.

El tipo de estudio característico del presente proyecto es predictivo, permitió establecer el comportamiento y la tendencia futura de las variables estratégicas internas y externas que afectan el desarrollo de las operaciones de la compañía Movitec Ltda., a partir de la identificación y análisis de la situación actual, generando así una planeación estratégica efectiva proyectada hacia el año 2023

A continuación se describen las fases que se tuvieron en cuenta para la aplicación de la metodología prospectiva: que las empresas del sector accedan de forma eficiente y transparente os, no probabil definir la planeaci Cundinamarcas. (escuel 


\section{Estrategia Organizacional}

Tabla 1. Fases metodológicas planeación estratégica prospectiva.

\begin{tabular}{|l|l|l|}
\hline \multicolumn{1}{|c|}{ FASE } & \multicolumn{1}{|c|}{ MÉTODO } & \multicolumn{1}{c|}{ INSTRUMENTOS } \\
\hline $\begin{array}{l}\text { Identificación de la situación actual } \\
\text { de la compañía Movitec Ltda. }\end{array}$ & Análisis estratégico & $\begin{array}{l}\text { Encuestas, entrevistas, } \\
\text { informes de gestión, estados } \\
\text { financieros de la compañía, } \\
\text { consulta de fuentes } \\
\text { secundarias externas }\end{array}$ \\
\hline $\begin{array}{l}\text { Determinación y clasificación de las } \\
\text { variables clave internas-externas }\end{array}$ & MICMAC & $\begin{array}{l}\text { Entrevistas aplicación software } \\
\text { MICMAC }\end{array}$ \\
\hline $\begin{array}{l}\text { IdentificaciEjes de Peter Schwartzles } \\
\text { y apuestaados y externas see en } \\
\text { la actualidad la compañ internos } \\
\text { y aprendizaje y crecimiento (Vidal, } \\
\begin{array}{l}\text { ón y análisis del juego de actores } \\
\text { involucrados }\end{array}\end{array}$ & MACTOR & $\begin{array}{l}\text { Entrevistas, aplicación } \\
\text { software MACTOR, encuestas }\end{array}$ \\
\hline $\begin{array}{l}\text { Determinación de escenarios } \\
\text { probables y apuesta }\end{array}$ & Ejes de Peter Schwartz & Cuestionario Delphi, entrevistas \\
\hline $\begin{array}{l}\text { Análisis y evaluación de acciones y } \\
\text { opciones estratégicas }\end{array}$ & $\begin{array}{l}\text { Importancia y } \\
\text { gobernabilidad IGO }\end{array}$ & $\begin{array}{l}\text { Entrevistas, aplicación matriz } \\
\text { IGO }\end{array}$ \\
\hline $\begin{array}{l}\text { Elaboración de planes de acción } \\
\text { Despliegue estratégico }\end{array}$ & Entrevistas \\
\hline
\end{tabular}

Fuente: elaboración propia

\section{RESULTADOS}

Con base en los resultados de la encuesta Delphi, y el análisis interno y externo de la compañía Movitec Ltda., se identificaron treinta variables estratégicas, las cuales se caracterizaron y calificaron según su grado de influencia y dependencia, mediante el método de Matriz de Impactos Cruzados Multiplicación Aplicada a una Clasificación (MICMAC) (Godet, 2009, p. 56).

De esta forma, se presenta a continuación el mapa de influencias y dependencias directas de las variables estratégicas: 


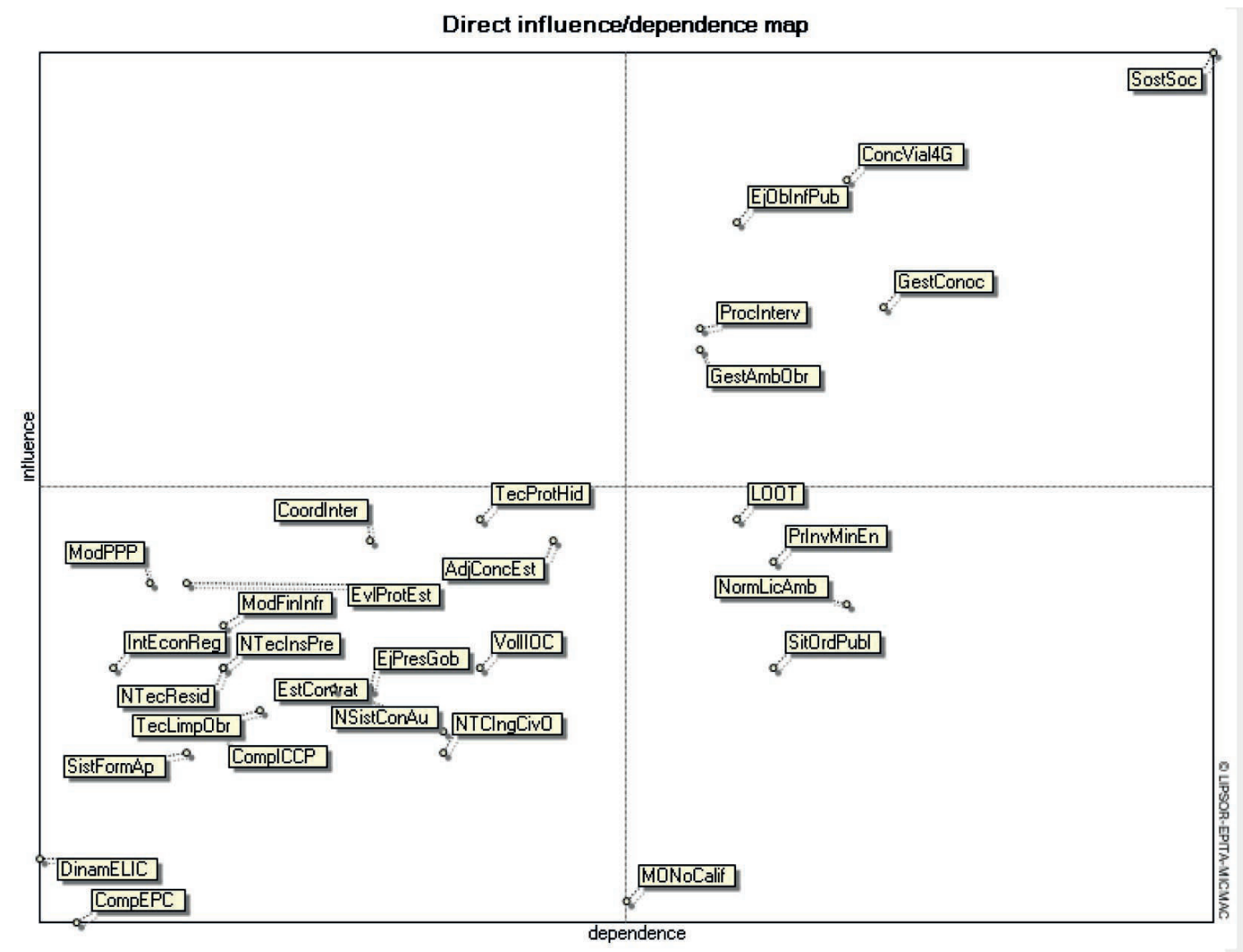

Figura 1. Mapa de influencia y dependencia directa variables estratégicas Movitec Ltda.

Fuente: elaboración propia a partir de Godet (2009)

Teniendo en cuenta la aplicación del método MICMAC, se pueden clasificar los factores de cambio o variables estratégicas que inciden directa e indirectamente en la gestión estratégica de la compañía Movitec Ltda. de la siguiente manera:

- Variables de enlace: para el caso de la compañía Movitec Ltda., las variables estratégicas de enlace o conflicto, corresponden al orden social -sostenibilidad social de los proyectos-, político-administrativo -concesiones viales de cuarta generación, procesos y procedimientos de interventoría de obras de infraestructura, y ejecución de obras de infraestructura pública-, científico-tecnológico -gestión del conocimiento en obras civiles- y ecológico - gestión 
ambiental de las obras de infraestructura-. Dichas variables, se convierten en grandes factores de cambio para la empresa, puesto que se relacionan directamente con el desarrollo de su objeto social -prestación de servicios de ingeniería civil de obras de infraestructura-.

- Variables resultantes: las variables de salida o resultantes que inciden en la compañía Movitec Ltda. son del orden normativo-jurídico - Ley Orgánica de Ordenamiento Territorial y Normatividad para Licencias Ambientales de obras de infraestructura-, económico -proyectos de inversión de empresas del sector minero-energético- y social - situación de orden público-.

- Variables excluidas: las variables excluidas o autónomas del sistema son de tipo económico -comportamiento de la estadísticas de producción de cemento, dinámica de las estadísticas de edificación de licencias de construcción, comportamiento del índice de costos de la producción pesada, modelos de financiación de infraestructura e integración económica regional-, científico-tecnológico -tecnologías limpias en la construcción de obras civiles, nuevas tecnologías de inspección y predicción de materiales y estructuras, nuevas tecnologías de evaluación, gestión y reutilización de residuos, nuevos sistemas de control automatizado de máquinas-, normativo-jurídico -normas técnicas colombianas de ingeniería civil y obras y estatuto de contratación estatal-, político-administrativo -ejecución presupuestal del Gobierno- y cultural - sistemas de formación y aprendizaje del personal no calificado-.

- Variables de pelotón: las variables de pelotón son del orden político-administrativo -adjudicación de concesiones por parte del Estado y coordinación interinstitucional con entidades del Estado-, ecológico -técnicas para la protección de fuentes hídricas-, económico - volatilidad del indicador de inversión real en obras civiles IIOC y empleo de mano de obra no calificada- ,científico-tecnológico -métodos de análisis, materiales y técnicas para evaluación y protección de estructuras-, social -modelos de participación público privada PPP-.

Por su parte, se identificaron 25 actores involucrados o asociados a las operaciones de Movitec Ltda., para los cuales se analizaron los objetivos y juegos asociados a cada uno de éstos, por medio de la aplicación del método de actores, objetivos y relaciones de fuerza MACTOR. A continuación se presenta el mapa de influencias y dependencias directas entre actores: 


\section{Direct influence/dependence map}

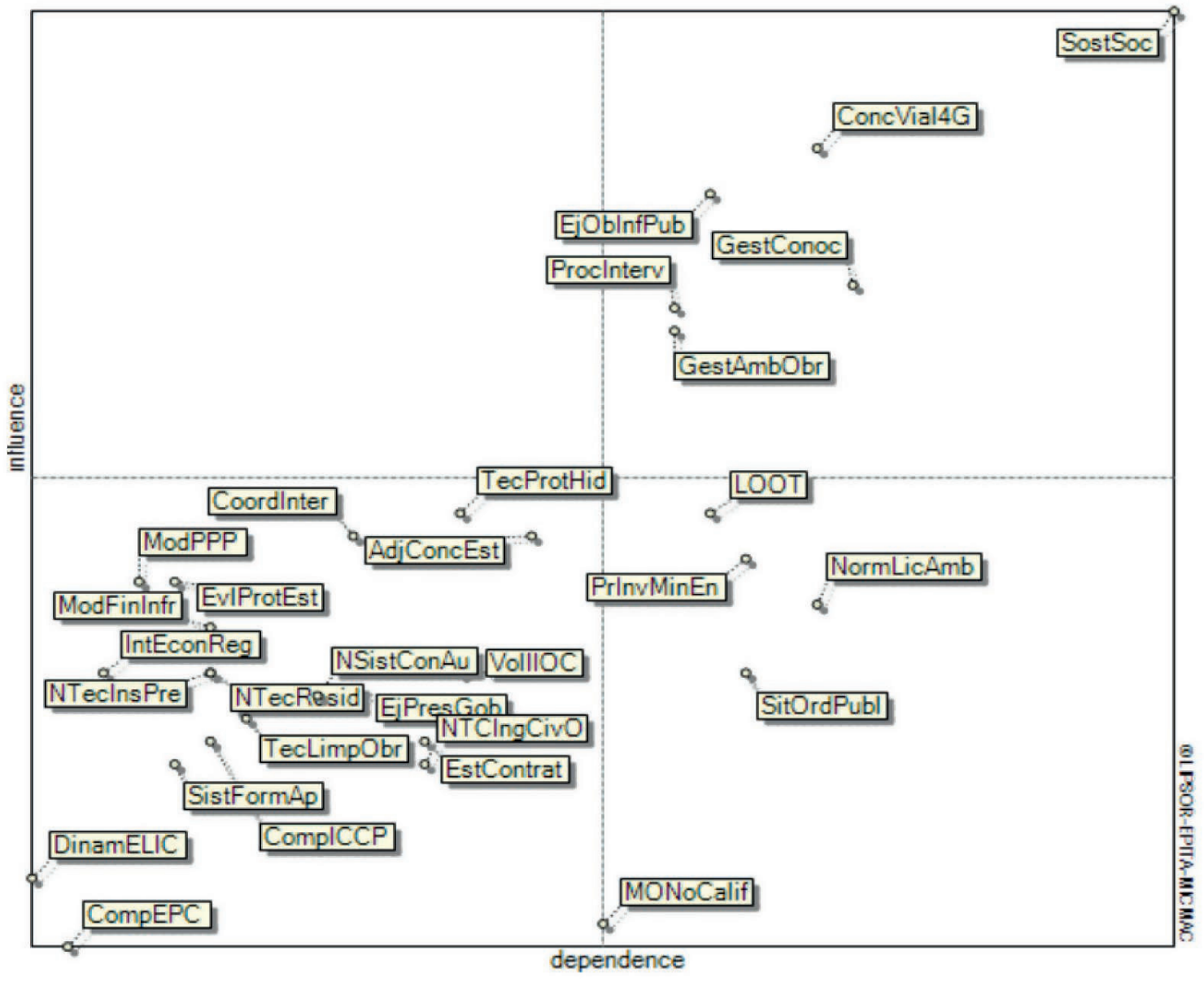

Figura 2. Mapa de influencias y dependencias directas entre actores.

Fuente: elaboración propia a partir de Godet,(2009)

De esta forma, la clasificación o tipología de juego del actor, según su grado de influencia y dependencia, se establece de la siguiente manera:

- Actores de enlace: los actores de enlace o de mediano poder (Mojica, 2008, p. 209), que se caracterizan por su alto grado de influencia y dependencia, corresponden a: gobierno Nacional, organizaciones civiles, competidores, Ministerio de Ambiente y Desarrollo Sostenible, Ministerio de Minas y Energía, Ministerio de Transporte, INVÍAS, Agencia Nacional de Infraestructura (ANI), Cámara Colombiana de Infraestructura, Socios, Clientes y Departamento Nacional de Planeación. 
- Actores dominantes: para la compañía Movitec Ltda., los actores dominantes o de alto poder (Mojica, 2008, p. 209), que debe tener muy en cuenta para la visualización y alcance de su escenario apuesta, en el año 2023, son: Servicio Nacional de Aprendizaje (SENA), Empresas Aseguradoras y Administradoras de Riesgos Laborales (ARL).

- Actores dominados: en este caso, los actores dominados o de bajo poder, cuyo grado dependencia es alto y la influencia es baja (Mojica, 2008, p. 209), corresponden a FINDETER, FONADE y proveedores.

- Actores autónomos: se caracterizan por su poca influencia y poca dependencia sobre los demás actores (Mojica, 2008, p. 209). Teniendo presente la Matriz de Influencia Directa entre actores (MID), se concluye que los actores autónomos o de muy bajo poder son: Sistema General de Regalías (SGR), ICONTEC, bolsas de empleo, DIAN, Sociedad Colombiana de Ingenieros SCI, CAMACOL y ACODAL.

Teniendo en cuenta las variables estratégicas se definieron los direccionadores o vectores de futuro, a fin de definir los escenarios, por medio del método de matriz de ejes cruzados de Peter Schwartz (Schwartz, 1996). Los dos grandes direccionadores o vectores de futuro están relacionados con el nivel de eficiencia y transparencia en la adjudicación y concesión de obras de infraestructura pública e ingeniería civil, por parte del Estado, por cuanto esta dinámica garantizaría la competitividad no solo de la empresa Movitec Ltda., sino también de las demás empresas competidoras del sector. Especialmente si se tiene presente que un sistema de concesión de obras eficiente y transparente dinamizará la inversión real en el subsector de obras civiles, y mejorará la infraestructura de las regiones y el país en general, en el marco de la entrada en vigencia de nuevos acuerdos comerciales y tratados de libre comercio TLC con otros países, regiones o bloques económicos.

Por su parte, el otro direccionador o vector de futuro está estrechamente ligado a la innovación tecnológica en materiales, máquinas, equipos, técnicas y métodos social y ambientalmente sostenibles, los cuales deben cumplir con altos estándares de calidad y garantizar la confiabilidad y conformidad de las obras de infraestructura pública que se lleven a cabo. De esta forma, las compañías dedicadas a la prestación de servicios de obras de ingeniería civil gestionarán sus operaciones y demás procesos administrativos, basados en la Responsabilidad Social Empresarial (RSE).

A continuación se presenta el mapa de ejes cruzados de Peter Schwartz, a fin de definir los escenarios: 


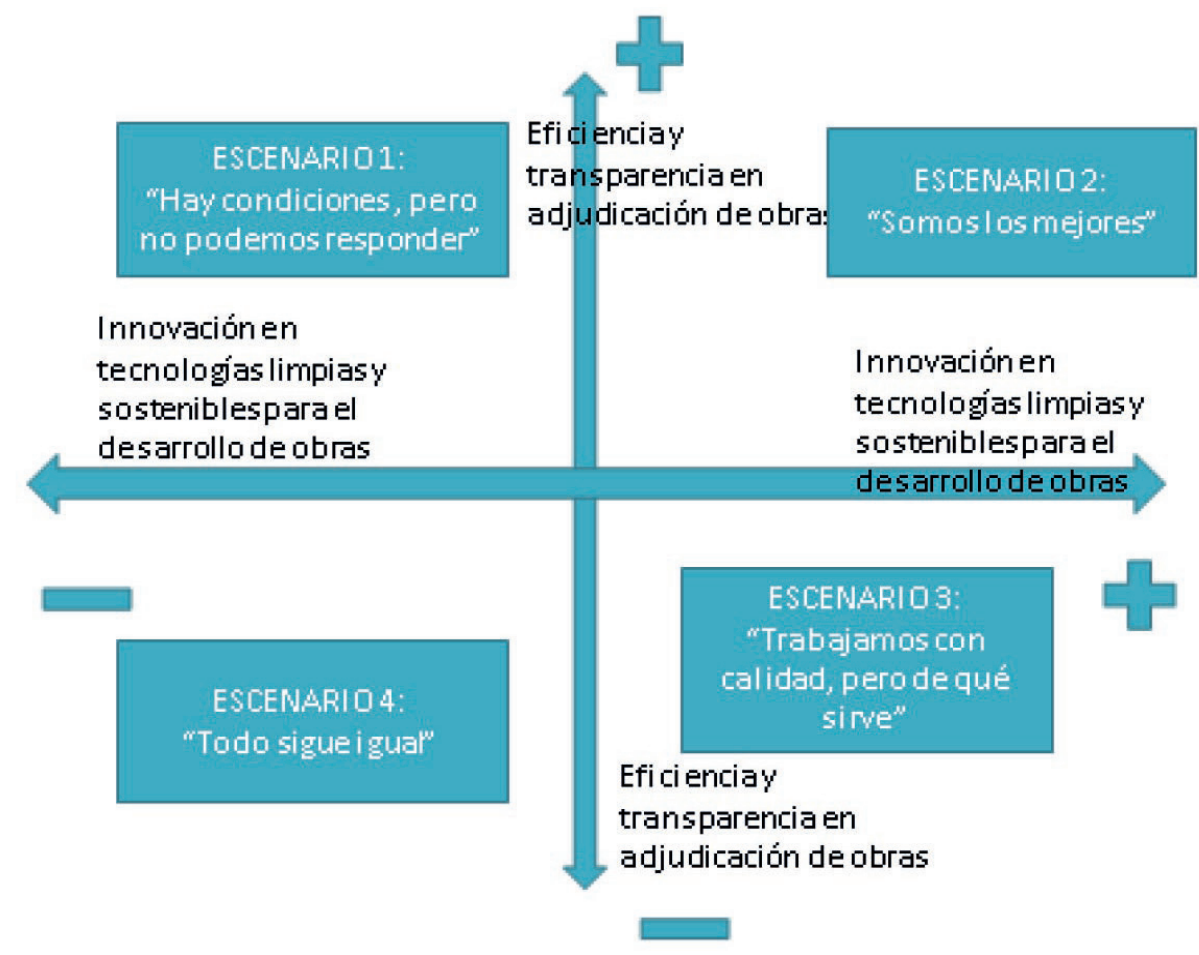

Figura 3. Mapa de ejes de Schwartz escenarios compañía Movitec Ltda.

Fuente: elaboración propia

Basados en el mapa de ejes de Schwartz, se define como escenario apuesta el escenario 2 denominado "Somos los mejores", en el que Movitec Ltda. será líder en el subsector de la construcción de obras civiles en Colombia. Esto se logrará por medio de una asociatividad estratégica importante con el Estado y las empresas del sector, a fin de aprovechar las condiciones favorables del gobierno Nacional en materia de contratación. Se fomentarán mayor número de integraciones verticales hacia delante -clientes y demás stakeholders o grupos de interés- y hacia atrás - proveedores-, con el propósito de garantizar calidad en las obras y servicios que desarrolle la compañía, a través de la utilización de tecnologías, técnicas y métodos social y ambientalmente sostenibles

Con el fin de alcanzar el escenario apuesta o futurible ideal para Movitec Ltda. se identificaron doce estrategias, las cuales se agruparon en perspectivas como financiera, clientes, procesos internos y aprendizaje y crecimiento. A continuación se define el mapa estratégico para la compañía en el año 2023, a fin de garantizar la implementación de dichos cursos de acción: 


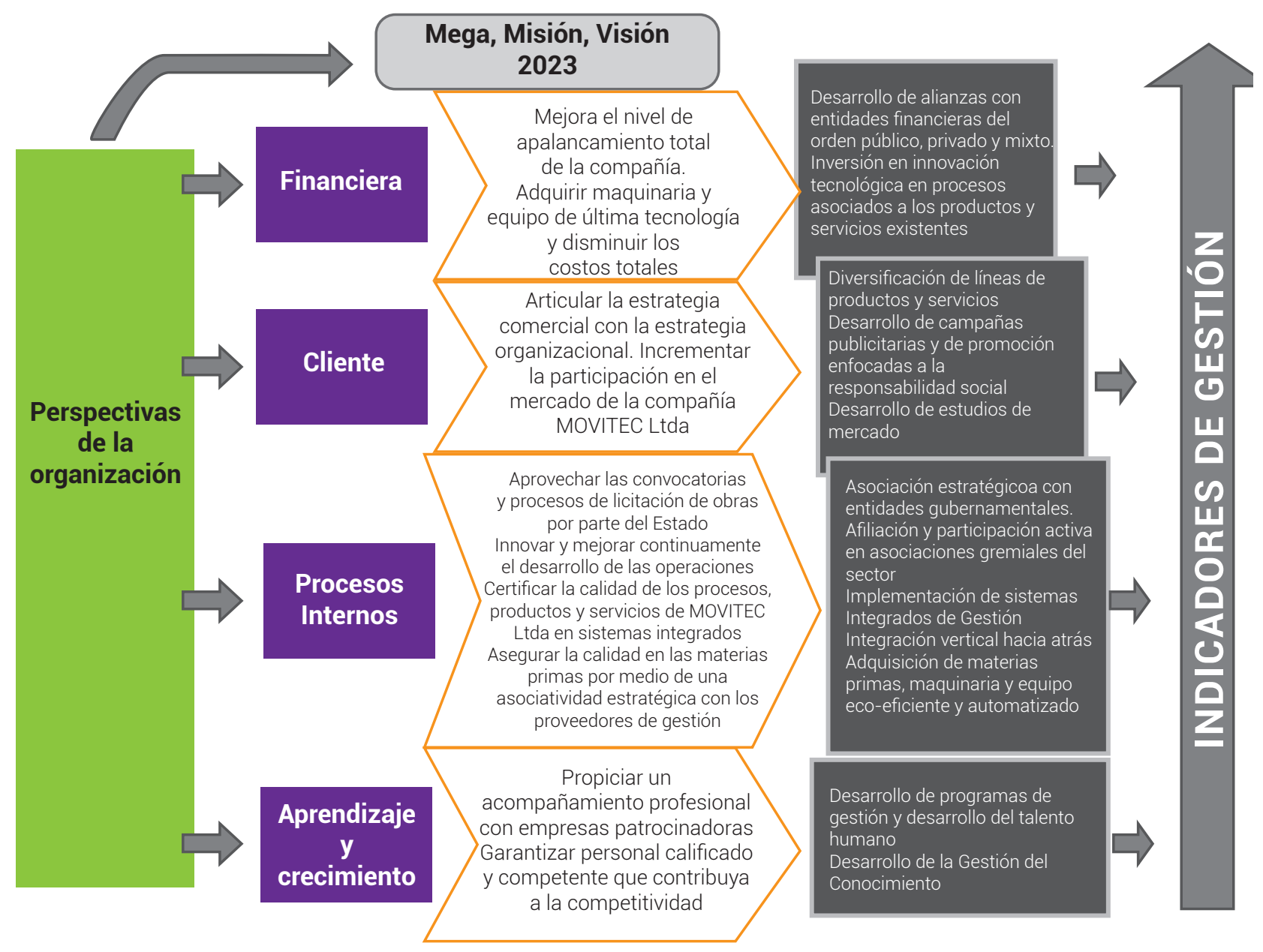

Figura 4. Mapa estratégico Movitec Ltda.

Fuente: elaboración propia

\section{CONCLUSIONES}

El presente estudio permitió identificar debilidades estratégicas de Movitec Ltda. en factores críticos de éxito como: el posicionamiento de los productos y servicios que ofrece, la innovación tecnológica en maquinaria y equipos sostenibles, la membresía y participación activa en entidades gremiales como la Cámara
Colombiana de Infraestructura y la certificación en Sistemas Integrados de Gestión, los cuales le permiten a la compañía ser más competitiva en el subsector de la construcción de obras de ingeniería civil. De esta forma, el compromiso de la gerencia general de la compañía permite visualizar el mejor escenario o futurible ideal para el año 2023, al cual Movitec Ltda. debe 
aspirar no solo para sobrevivir en el mercado, sino para constituirse en la empresa líder en el subsector.

Es importante que la empresa Movitec Ltda. continúe con el proceso de desarrollo y crecimiento colectivo en la línea del tiempo, y comprometa al talento humano colaborador en la constante renovación de sus proyectos, y la anticipación a los riesgos que se puedan presentar, por medio de la implementación de estrategias de crecimiento comercial y políticas empresariales sostenibles.

Movitec Ltda. debe generar el aumento de las capacidades de aprendizaje, medidas a través de la planeación estratégica e implementar herramientas de planificación y mejora, con el objetivo de asegurar el éxito de sus proyectos. Todo lo anterior debe estar apoyado en elementos críticos como el factor humano, la dirección y apoyo de la gerencia, y hacer parte vital de la aplicación de modelos que permitan medir la eficiencia de las estrategias operativas en cada uno de los proyectos.

De modo que, a través de la planeación estratégica prospectiva, la empresa puede tener presente los siguientes riesgos operacionales y de mercado, que pueden limitar o inhibir el logro de sus objetivos estratégicos y su escenario apuesta:
- Deficiencia en la definición de las metas a obtener: se presenta cuando el personal no tiene claras las estrategias de la empresa, ni cuáles son las medidas a tomar.

- Definición de la visión a futuro: el personal asume que si cumple los procesos y procedimientos es suficiente para lograr los objetivos propuestos.

- Costos: generar más gastos de los permitidos en la implementación de modelos prospectivos conlleva a costos elevados.

- Planeación: es importante dejar claros los límites entre los objetivos de la organización y las estrategias para su obtención

Por tanto, se recomienda a Movitec Ltda. buscar siempre el balance entre las estrategias definidas y el seguimiento de los indicadores de gestión, por medio de una estructuración organizacional basada en procesos, y la aplicación efectiva de los principios centrados en la estrategia y el escenario apuesta, los cuales garantizan un óptimo posicionamiento de los productos y servicios; y por consiguiente, el liderazgo competitivo en el subsector de la construcción de obras de ingeniería civil.

Como en todo emprendimiento existen riesgos y es necesario anticiparse a los mismos, la 
empresa debe desarrollar un programa de gestión del conocimiento, el cual se involucre en todos sus procesos. Así como aumentar y mejorar la infraestructura tecnológica, implementar innovación tecnológica para conservar la información de los clientes como sus requisitos, sus exigencias y medio de desarrollo, establecer acuerdos y alianzas de tercerización de servicios con empresas del sector, afiliarse y participar activamente en entidades gremiales, y desarrollar alianzas con entidades públicas, privadas y mixtas para la obtención de recursos y contratos de obras de infraestructura pública, aprovechando la coyuntura de los planes de desarrollo y las megaobras propuestas para los próximos diez años.

Como el escenario apuesta implicaría una innovación en tecnologías limpias, ecoeficientes, sostenibles, y una mejor eficiencia y transparencia en la adjudicación de obras y contratos, se debe empoderar no solo a la alta dirección, sino a los demás colaboradores de Movitec Ltda., a fin de que se anticipen a los cambios tecnológicos en el subsector de la construcción de obras civiles, mejorando el desarrollo de las operaciones en cuanto a eficiencia, eficacia, efectividad, productividad y minimización de costos operacionales

\section{REFERENCIAS}

Alvarado, Duque y Hoyos. (2011). Guía para la aplicación de un Modelo de Planeación Estratégica
Prospectiva Integral para Finagro. Bogotá, Colombia: Universidad de La Sabana. En línea. Recuperado de: http://intellectum.unisabana. edu.co:8080/jspui/bitstream/10818/166/1/ Yolanda\%20Alvarado\%20Miranda.pdf.

Arce, D. (2003). El Contrato de Obra, razones de las órdenes de cambio o reclamaciones de los contratistas. Bogotá, Colombia: Pontificia Universidad Javeriana. En línea. Recuperado de: http:// www.javeriana.edu.co/juridicas/pub_rev/documents/281-296.pdf

Asociación Española de Fabricantes de Maquinaria de Construcción, Obras Públicas y Minería (ANPOMYC). (2011). Estudio de Tendencias Tecnológicas en el Sector de Maquinaria de Obras Públicas, Construcción y Minería. En línea. Recuperado de: http://www.anmopyc.es/ resources/archivosCkeditor/files/Estudio\%20 Tendencias\%20Tecnologicas\%20MOPYC.pdf

Congreso de Colombia. (1993). Ley 80 de 1993: Por la cual se expide el Estatuto General de la Contratación de la Administración Pública. Bogotá, Colombia: Congreso de Colombia. En línea. Recuperado de: http://www.saludcapital.gov.co/DJC/Normatividad\%20Aplicable/ Ley_80_1993.pdf.

Congreso de Colombia. (2007). Ley 1150 de 2007: Por medio del cual se introducen medidas para 
la eficiencia y transparencia en la Ley 80 de 1993 y se dictan otras disposiciones generales sobre la contratación con Recursos Públicos. Bogotá, Colombia: Congreso de Colombia. En línea. Recuperado de: http://www.alcaldiabogota. gov.co/sisjur/normas/Norma1.jsp?i=25678

Diario El Colombiano (2013). La construcción crecerá en 2013 más que minería y petróleo: Anif. En línea. Recuperado de: http://www.elcolombiano. com/BancoConocimiento/L/la_construccion_ crecera_en_2013_mas_que_mineria_y_petroleo_anif/la_construccion_crecera_en_2013_ mas_que_mineria_y_petroleo_anif.asp

Fundación OPTI. (2010). Obra Civil: Estudio de Prospectiva del Sector Obra Civil en Construcción. En línea. Recuperado de: http://www.google. com.co/url?sa=t\&rct=j\&q=\&esrc=s\&source=we$b \& c d=4 \& v e d=0 C D E Q F j A D \& u r l=h t t p \% 3 A \%-$ 2F\%2Fwww.cgee.org.br\%2Fatividades\%2FredirKori\%2F2272\&ei=p47vU5ajH5C0yASgp4LgCA\&usg=AFQjCNHcMJtMsqlqmSVTNshNuUz9ei6JzQ\&sig2=Xa3HaGY6w26eAhHa7AvZJw\&bvm=bv.73231344,d.aWw

Godet, M. (2007). Prospectiva Estratégica: Problemas y Métodos. Paris, Francia: LIPSOR.

Godet, M. (2009). La Prospectiva Estratégica para las empresas y los territorios. Paris, Francia: LIPSOR.
Granados, H. y Arango, J. C. (2013). Estudio Prospectivo Del Hábitat y La Construcción En Colombia. Medellín, Colombia: Centro de Investigación y Prospectiva Institución Universitaria ESUMER. En línea. Recuperado de: http://es.calameo. com/read/000325005cb12cb9b0f42.

Hernández, R.; Fernández, C. y Baptista, P. (2010). Metodología de la Investigación. Ciudad de México, México: Mc Graw Hill.

Instituto Nacional de Formación Técnico Profesional (INFOTEP). (2013). Estudio Prospectivo de la Formación Profesional en el Sector de la Construcción Civil. Santo Domingo, República Dominicana: INFOTEP. En línea. Recuperado de: http://www.oitcinterfor.org/sites/default/files/ file_publicacion/Estudio\%20prospectivo $\% 20$ INFOTEP.pdf.

Mellicer, T. (2001). El Sector de la Construcción: Una Perspectiva Internacional. Valencia , España : Universidad Politécnica de Valencia. En línea. Recuperado de: http://books.google.com. co/books?id=EnWkcmTu2U0C\&pg=PA19\&I$\mathrm{pg}=\mathrm{PA} 19 \& \mathrm{dq}=$ prospectiva+estrategica $+\mathrm{sec}-$ tor+construccion \&source=bl\&ots $=h W R f 3 d-$ hw4P\&sig=-u8VG5FX2t9lay 9vbD29PiZJUYE\&hl=es\&sa=X\&ei=PkOSU9GOGoKnkQeX-

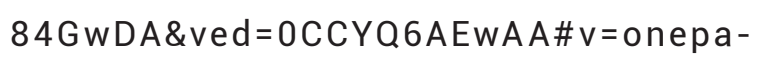
ge\&q=prospectiva\%20estrategica\%20sector $\% 20$ construccion\&f=false 
Miklos, T. y Arroyo, M. (2008). Prospectiva y Escenarios para el Cambio Social. Ciudad de México, México: Limusa.

Mintzberg, H.; Ahlstrand, B. y Lampel, J. (2010). Safari a la Estrategia: Una visita guiada por la jungla del management estratégico. Buenos Aires, Argentina: Granica S.A.

Mojica, F. (2008). La Construcción del Futuro: Concepto y modelo de prospectiva estratégica, territorial y tecnológica. Bogotá, Colombia: Universidad Externado de Colombia.

Movitec Ltda. (2012). Estados Financieros Consolidados años 2007-2011. Movitec Ltda.

Movitec Ltda. (2013). Informe de Gestión 2012. Movitec Ltda.

Porter, M. (2006). Estrategia y Ventaja Competitiva. Buenos Aires, Argentina: Ediciones Deusto.
Sallenave, J. P. (2002). Gerencia y Planeación Estratégica. Bogotá, Colombia: Editorial Norma.

Schwartz, P. (1996). The art of the long view. En línea. Recuperado de: http://www.ethicaladvisor.com/ pdf/books/THE_ART_OF_THE_LONG_VIEW.pdfSerna Gómez, H. (2014). Gerencia Estratégica: Teoría-Metodología-Mapas Estratégicos- Índices de Gestión- Alineamiento Ejecución Estratégica. Bogotá, Colombia: 3R Editores.

Universidad Nacional Abierta y a Distancia (UNAD) (2012). Protocolo de presentación de trabajos de grado programas de posgrado. Bogotá, Colombia: Escuela de Ciencias Administrativas, Contables, Económicas y de Negocios ECACEN.

Vidal Arizabaleta, E. (2005). Diagnóstico Organizacional: Evaluación sistémica del desempeño empresarial en la era digital. Bogotá, Colombia: ECOE Ediciones. 